\title{
Application of Circulating Tumor DNA in Early Detection of Breast Cancer
}

Reza Heidari ${ }^{1,{ }^{*}}$, Mostafa Akbariqomi ${ }^{1}$, Sattar Akbari Nakhjavani ${ }^{1}$, Hamid Kooshki ${ }^{1}$, Jamshid Motaei ${ }^{2}$, Mahdi Mortazavy ${ }^{3}$, Shilan Shafie ${ }^{1}$, Moloud Absalan ${ }^{1}$, Paria Sadat Lavasani ${ }^{1}$, Naser Samadi ${ }^{4}$

${ }^{1}$ Department of Molecular Medicine, School of Advanced Technologies in Medicine, Tehran University of Medical Sciences, Tehran, Iran

${ }^{2}$ Department of Medical Genetics, Mashhad University of Medical Sciences, Mashhad, Iran.

${ }^{3}$ Department of Molecular Genetics, National Institute of Genetic Engineering and Biotechnology, Tehran, Iran

${ }^{4}$ Department of Bacteriology and Virology, Shiraz Medical School, Shiraz University of Medical Sciences, Shiraz, Iran

* Corresponding Author: Reza Heidari, Department of Molecular Medicine, School of Advanced Technologies in Medicine, Tehran University of Medical Sciences, Tehran,

DOI: $10.21859 /$ mci-supp-19 Iran.E-mail: Nipt.2015@yahoo.com

\section{Keywords:}

Circulating Tumor DNA

Breast Cancer

Early Detection

Epigenetic Marker

\begin{abstract}
Introduction Circulating tumor DNA (ctDNA) as a non-invasive marker that can provide more information on genetic and epigenetic alterations in tumor cells. The epigenetic modifications occur in the early stages of cancer, thus, it is considered as a target for early detection and prevention. The current study is to develop a ctDNA-probe based technology using capture, enrichment and analysis of epigenetic markers for early detection of breast cancer (BC).

Materials and Methods: ctDNA from 45 women with early stage breast cancer and 90 healthy women as control samples were isolated. Methylation analysis performed for 6 differential regions by MeDIP-Probe method and all of them were sequenced. The KLF9 gene used as an internal control for amplifying the regions despite Methylation status. Results: The methylation analysis presented significant differences in methylation of two regions RASSF1A and HOXA10 between BC patients and healthy samples. Methylation in the regions was higher in $\mathrm{BC}$ than control case $(\mathrm{P}<0.001)$.

Conclusions: Combinations of multiple methylation regions or $\mathrm{CpG}$ Island improved the positive predictive value for breast cancer detection. Analysis of methylation pattern of ctDNA using probe-base technology with screening methods such as mammography in high-risk women leads to noninvasive early detection of breast cancer.
\end{abstract}

